\title{
REPRESENTING MEASURES AND TOPOLOGICAL TYPE OF FINITE BORDERED RIEMANN SURFACES(1)
}

\author{
BY \\ DAVID NASH
}

\begin{abstract}
A finite bordered Riemann surface $\Re$ with $s$ boundary components and interior genus $g$ has first Betti number $r=2 g+s-1$. Let $a$ be any interior point of $\Re$ and $e_{a}$ denote evaluation at $a$ on the usual hypo-Dirichlet algebra associated with $\mathscr{q}$. We establish some connections between the topological and, more strongly, the conformal type of $\Re$ and the geometry of $\mathfrak{R}_{a}$, the set of representing measures for $e_{a}$. For example, we show that if $\mathfrak{R}_{a}$ has an isolated extreme point, then $\Re$ must be a planar surface. Several questions posed by Sarason are answered through exhausting the possibilities for the case $r=2$.
\end{abstract}

1. Let $R$ be a finite open Riemann surface with boundary $\partial R$ consisting of a finite nonzero number $s$ of disjoint Jordan curves (see Ahlfors and Sario [4, p. 117]). The first Betti number of $R$, which we denote by $r$, equals $2 g+s-1$, where $g$ is the interior genus of $R$. We take $\partial R$ to be oriented with the induced orientation from $R$. Let $A$ be the hypo-Dirichlet algebra (see [2], [15]) of functions continuous on $\partial R$ and possessing continuous extensions to $\bar{R}=R \cup \partial R$ which are holomorphic on $R$. Fix a point $a \in R$ and let $e_{a}$ denote evaluation on $A$ at $a$; i.e., $e_{a}(f)=f(a)$ for $f \in A$. Let $\Re_{a}$ be the compact convex set of representing measures for $e_{a}$. A measure $m \in \mathfrak{N}_{a}$ is a positive Borel measure of total mass one supported on $\partial R$ such that $f(a)=\int_{\partial R} f d m$ for all $f \in A$.

In this paper we shall show some connections between the topological and, more strongly, the conformal type of $R$ and the geometry of $\pi_{a}$. We will let $S^{2}$ denote the Riemann sphere and $R e$ be the real axis. The set $\pi_{a}$ will be called strictly convex if $\partial \Re_{a}$ consists entirely of extreme points. The principal results are stated below.

Theorem 1.1. If $\mathfrak{T}_{a}$ has an isolated extreme point, then $R$ is conformally equivalent to $S^{2}$ minus a finite number of slits contained in $\mathrm{Re}$.

The next theorem exhausts the possibilities for $\mathfrak{\Re}_{a}$ when $r=2$, and thus answers Questions 1 and 3 on p. 376 of [12].

Received by the editors August 20, 1972.

AMS (MOS) subject classifications (1970). Primary 30A46, 46J10; Secondary 30A52.

Key words and phrases. Finite bordered Riemann surface, representing measure, real annihilating measure, hypo-Dirichlet algebra, convex body, extreme point, Betti number, interior genus, conformal equivalence.

(1) This paper is based on a portion of the author's doctoral dissertation submitted at the University of California at Berkeley. The author wishes to express appreciation to his adviser, Professor Donald Sarason, for his encouragement and helpful suggestions and to the referee of an earlier version of this paper for a comment which led to an improvement in the construction of the conformal map in Theorem 1.1. 
Theorem 1.2. If $g=0$ and $s=3$, then $\mathfrak{R}_{a}$ has precisely four extreme points if $a$ lies on one of three distinguished analytic arcs, and $\mathfrak{R}_{a}$ is strictly convex if a lies off these arcs. If $g=s=1$, then $\Re_{a}$ is strictly convex for all $a \in R$.

The geometry of $\mathfrak{R}_{a}$ is not yet completely known for all $R$ 's with $r>2$, but some results are presented for particular cases. Other relevant material can be found in [12].

The proofs given are of an elementary nature and rely primarily on properties of zero sets of representing measures.

2. We shall begin with some basic facts about $\mathfrak{N}_{a}$ and $V$, the space of real annihilating measures of $A$.

Let $m_{a}$ denote harmonic measure for $e_{a}$. This measure has the representation $d m_{a}=* d_{p} g(p, a) /-2 \pi$ on $\partial R$, where $g(p, a)$ is the Green's function for $\bar{R}$ with singularity at $a, d_{p}$ is exterior differentiation on $g(p, a)$ as a function of $p$ and $*$ is the Hodge star operator (see [13, pp. 217-218]). Fix a nowhere vanishing abelian differential $\omega$ which is holomorphic on $\bar{R}$. Then there is a function $Q_{m_{a}}$ which is holomorphic on $\partial R$ such that $* d_{p} g(p, a) /-2 \pi=Q_{m_{a}} \omega$ where defined. If $m$ is any element of $\pi_{a}$, then $m-m_{a} \in V$. The space $V$ is $r$ dimensional and is spanned by Schottky differentials $\omega_{1}, \ldots, \omega_{n}$ which are real on $\partial R$. Thus $d m=Q_{m_{a}} \omega$ $+\sum_{i=1}^{r} a_{i} \omega_{i}$ for some real numbers $a_{i}$. It follows that there is a function $Q_{m}$ which is holomorphic on $\partial R$ such that $d m=Q_{m} \omega$. From the known structure of $* d_{p} g(p, a)$ and of the $\omega_{i}$ 's, it can be seen that $Q_{m}$ can be regarded as a meromorphic differential on $\bar{R}$ whose only pole is a simple pole at $a$.

Since $Q_{m} \omega$ is real on $\partial R$, it can be extended by the reflection principle to a meromorphic differential on $\hat{R}$, the (compact) doubled Riemann surface associated with $R$. The sum of the orders of the zeros minus the sum of the orders of the poles of a nontrivial meromorphic differential on a compact Riemann surface is $2 G-2$, where $G$ is the genus of the surface (see [13, p. 267]). The genus of $\hat{R}$ is $r$, the first Betti number of $R$, so if $Q_{m} \omega$ extended to $R$ has $p$ zeros on $R$ and $q$ zeros on $\partial R$, counting multiplicities, then $Q_{m} \omega$ has $2 p+q$ zeros on $R$, which along with tws simple poles on $R$ implies $2 r-2=2 p-q-2$. We have established

Theorem 2.1. $Q_{m}$ has $r$ zeros on $\bar{R}$ provided zeros on $\partial R$ are counted with one-half their multiplicities,

Definition 2.2. Let $Z\left(Q_{m}\right)$ denote the set of zeros of $Q_{m}$ in $\bar{R}$. For convenience we shall identify $m$ and $Q_{m} \omega$ and call $Z\left(Q_{m}\right)$ the zero set of $m$.

In the case of harmonic measure, $d m_{a}=* d_{p} g(p, a) /-2 \pi=Q_{m_{a}} \omega$, it happens that $* d_{p} g(p, a)$ is never zero on $\partial R$; therefore, $Q_{m_{e}}$ has no zeros on $\partial R$. We have shown

Theorem 2.3. The measure $m_{a}$ has $r$ zeros on $R$.

Theorem 2.4. If $m \in \mathfrak{R}_{a}$ and $d m=Q_{m} \omega$ and $p \in Z\left(Q_{m}\right) \cap \partial R$, then $p$ has even multiplicity. 
Proof. The conclusion follows from the fact that $d m=Q_{m} \omega$ is nonnegative on $\partial R$.

An argument similar to the one used to prove Theorem 2.1 establishes the following.

Theorem 2.5. Each nonzero $\eta \in V$ has $r-1$ zeros on $\bar{R}$ provided zeros on $\partial R$ are counted with one-half their multiplicities.

If the elements of $\mathfrak{N}_{a}$ are regarded as meromorphic differentials on $\bar{R}$, then it is clear that $\partial \Re_{a}$ consists precisely of those elements of $\mathfrak{R}_{a}$ with zeros on $\partial R$.

3. The following, through Lemma 3.1, paraphrases material in Ahern [1]. If $P$ is an $r-1$ dimensional hyperplane in the vector space of Schottky differentials on $\bar{R}$ such that $\mathfrak{N}_{a} \cap P=\partial \mathfrak{N}_{a} \cap P$ and is not empty, then $P$ is called a supporting hyperplane. If the interior of $\partial \Re_{a} \cap P$ is not empty, then this interior is called a face of $\pi_{a}$. If $m$ belongs to a face, then there is a unique supporting hyperplane containing $m$. If $m_{1}$ and $m_{2} \in \Re_{a}$, we define

$$
\left[m_{1}, m_{2}\right]=\left\{\lambda m_{1}+(1-\lambda) m_{2} \mid 0 \leq \lambda \leq 1\right\} .
$$

Lemma 3.1. Suppose $\mathfrak{T}_{a}$ has faces $F_{1}$ and $F_{2}$, and $m_{1} \in F_{1}$ and $m_{2} \in F_{2}$. Then $F_{1}=F_{2}$ if and only if $\left[m_{1}, m_{2}\right] \subset \partial \Re_{a}$.

Proof. If $F_{1}=F_{2}$, then it is obvious that $\left[m_{1}, m_{2}\right] \subset \partial \Re_{a}$. If $\left[m_{1}, m_{2}\right] \subset \partial \Re_{a}$, then by $\left[6\right.$, p. 5] there is a supporting hyperplane $P$ which contains $m_{1}$ and $m_{2}$. Since $m_{1}$ and $m_{2} \in P$ and $m_{1}$ and $m_{2} \in F_{2}$, the uniqueness property of supporting hyperplanes implies the interior of $P \cap \partial \Re_{a}=F_{1}=F_{2}$.

Let $C_{1}, \ldots, C_{s}$ be the components of $\partial R$ and $\phi_{j}(p)=r_{j} \exp \left(i \theta_{j}\right)$ be a uniformizer which maps a neighborhood of $C_{j} \subset R$ onto a neighborhood of the unit circle $T$ in $\mathbf{C}$ such that $\phi_{j}\left(C_{j}\right)=T, 1 \leq j \leq s$. Each $\mu \in \Re_{a}$ is represented on $T$ by $\left(d \mu / d \theta_{j}\right)\left(\theta_{j}\right) d \theta_{j}$, where $\left(d \mu / d \theta_{j}\right)\left(\theta_{j}\right) \geq 0$ and is analytic. Similarly, each $\eta \in V$ is represented on $T$ by $\left(d \eta / d \theta_{j}\right)\left(\theta_{j}\right) d \theta_{j}$, where $\left(d \eta / d \theta_{j}\right)\left(\theta_{j}\right)$ is real and analytic.

Lemma 3.2. A measure $\mu \in \partial \Re_{a}$ is not an extreme point if and only if there is an $\eta \in V$ such that each zero of $\mu$ on $\partial R$ is covered by a zero of $\eta$ with at least the same multiplicity.

Proof. If $\eta \in V$ satisfies the condition stated, then $\mu+\varepsilon \eta \in \partial \Re_{a}$ for all $\varepsilon$ in some sufficiently small neighborhood of 0 , and $\mu$ fails to be extreme.

If $\mu$ is not extreme, then $\mu=\left(\mu_{1}+\mu_{2}\right) / 2$ for some $\mu_{1} \neq \mu_{2} \in \partial \Re_{a}$. The functions $d \mu_{1} / d \theta_{j}$ and $d \mu_{2} / d \theta_{j}, 1 \leq j \leq s$, are nonnegative on $T$; therefore, each zero of $\mu$ on $\partial R$ is a zero of $\mu_{1}$ and $\mu_{2}$ with at least as high multiplicity. The measure $\eta=\mu_{1}-\mu_{2}$ satisfies the conclusion.

Corollary 3.3. If $\mu \in \mathfrak{N}_{a}$ has all its zeros on $\partial R$, then $\mu$ is extreme.

Proof. If not, there is an $\eta \in V$ with $r$ zeros on $\partial R$. This contradicts 2.5. 
Lemma 3.4. Let $K \subset \partial \Re_{a}$ be a nonempty convex set. Then there is $a P \in \partial R$ such that $\mu(P)=0$ for all $\mu \in K$.

Proof. Fix some $m \in K$ and let $P_{1}, \ldots, P_{n}, 1 \leq n \leq r$, be the zero points of $m$ on $\partial R$. Suppose for each $P_{i}$ there is some $\mu_{i} \in K$ such that $\mu_{i}\left(P_{i}\right) \neq 0$. Then the measure $\mu$ defined by $\left(\mu_{1}+\cdots+\mu_{n}\right) / n$ is in $K$ and has no zeros in common with $m$. It follows that $(\mu+m) / 2$ has no zeros on $\partial R$. But this is impossible since $(\mu+m) / 2 \in K \subset \partial \mathfrak{T}_{a}$. We conclude that for some $P_{j}, 1 \leq j \leq n, \mu\left(P_{j}\right)=0$ for all $\mu \in K$.

We shall call $K \subset \partial \Re_{a}$ a maximal convex subset of $\partial \Re_{a}$ if it is convex and is not properly contained in any convex subset of $\partial \Re_{a}$.

Lemma 3.5. Let $m \in \partial \Re_{a}$ and $Z=\left\{P_{1}, \ldots, P_{n}\right\}, 1 \leq n \leq r$, be the zeros of $m$ on $\partial R$. Then $m$ is contained in at most $n$ distinct maximal convex subsets of $\partial \pi_{a}$.

Proof. Suppose $K_{1}$ and $K_{2}$ are distinct maximal convex subsets of $\partial \Re_{a}$ which contain $m$. Then by Lemma 3.4 for some $P_{i}, P_{j} \in Z$ we have $\mu_{1}\left(P_{i}\right)=0$ for all $\mu_{1} \in K_{1}$ and $\mu_{2}\left(P_{j}\right)=0$ for all $\mu_{2} \in K_{2}$. We claim that $P_{i} \neq P_{j}$. Suppose $P_{i}=P_{j}$ and fix some $\mu \in K_{2} \backslash K_{1}$. Then the convex set $C=\left\{\lambda \mu_{1}+(1-\lambda) \mu \mid \mu_{1} \in K_{1}, 0\right.$ $\leq \lambda \leq 1\}$ (the convex hull of $K_{1} \cup\{\mu\}$ ) is contained in $\partial \Re_{a}$ since all measures in $C$ vanish at $P_{i}$. But $K_{1} \subseteq C$, a contradiction. Thus $P_{i} \neq P_{j}$ and the desired conclusion follows.

Lemma 3.6. If the boundary of an $n$ dimensional compact convex body has at most $n+1$ maximal convex subsets, then the convex body is an $n$-simplex.

Proof. The lemma is trivially true for $n=0$. Assume it is true for $n=k$. Let $K$ be a $k+1$ dimensional compact convex body with boundary containing at most $k+2$ maximal convex subsets. Denote these subsets by $F_{1}, \ldots, F_{j}$, where $j \leq k+2$. Let $H$ be a hyperplane which contains an interior point of $K$ but which does not intersect $F_{1}$. Then $H \cap K$ is a $k$ dimensional compact convex body. Each maximal convex subset of $\partial(H \cap K)$ is the intersection of $\partial(H \cap K)$ with some $F_{i}, 2 \leq i \leq j$; therefore, by the induction hypothesis, $H \cap K$ is a $k$ simplex. Since a $k$-simplex has $k+1$ faces, it follows that $j=k+2$ and that the faces of $H \cap K$ are $H \cap K \cap F_{i}, 2 \leq i \leq k+2$. By the preceding it is clear that for any hyperplane $H$ containing an interior point of $K$ but disjoint from $F_{1}$, the faces of $\partial(H \cap K)$ are $H \cap K \cap F_{i}, 2 \leq i \leq k+2$. This implies that each $F_{i}, 2 \leq i \leq k+2$, is $k$ dimensional. By a similar argument, using hyperplanes disjoint from $F_{2}$ and passing through interior points of $K$, we can conclude $F_{1}$ is also $k$ dimensional.

Now let $H_{i}$ denote the supporting hyperplane of $F_{i}, 1 \leq i \leq k+2$. Each $H_{i}$ is the boundary of a closed half space $S_{i}$ such that the interior of $S_{i} \cap K$ is nonempty. Introduce coordinates $x=\left(x_{1}, \ldots, x_{r}\right)$ so that $S_{1}=\left\{x \mid x_{1} \geq 0\right\}$. Let $H(t)$ denote the hyperplane $\left\{x \mid x_{1}=t\right\}$. Then $H(0)=H_{1}$. Let $t_{0}=$ $\sup \{t \mid H(t) \cap K$ is nonempty $\}$. Since $K$ is $k+1$ dimensional and compact, 
$0<t_{0}<\infty$. Let $S=S_{1} \cap \cdots \cap S_{k+2}$. Then $K \subset S$. By the first paragraph, for any $t$ such that $0<t<t_{0}$, we have $H(t) \cap K=H(t) \cap S$. If $H(0) \cap K$ $\neq H(0) \cap S$, then the same inequality would hold for sufficiently small $t>0$, a contradiction. Hence $H(0) \cap S=H(0) \cap K=F_{1}$, and $F_{1}$ is a face of $S$. Similarly, $F_{j}$ for $2 \leq j \leq k+2$ is a face of $S$; consequently, $\partial S \subset \partial K$. Since $K \subset S$, it follows that $K=S$, a $(k+1)$-simplex.

Lemma 3.7 If $\mu$ is an isolated extreme point of $\mathfrak{T}_{a}$, then $\mu$ has $r$ distinct double zeros on $\partial R$, and there is an $m \in \Re_{a}$ with $r-1$ double zeros on $\partial R$ and one simple zero in $R$.

Proof. Since $\mu$ is isolated, there is a closed half space $S$ such that $\mu$ is the only extreme point of $\pi_{a}$ in $\pi_{a} \cap S$, and $\mu \notin$ the hyperplane $\partial S$. We claim that if $m^{\prime} \in \partial \Re_{a} \cap S$ and $C$ is a maximal convex subset of $\partial \Re_{a}$ which contains $m^{\prime}$, then any extreme point of $C$ is extreme in $\pi_{a}$. To establish the claim let $m_{1}$ be extreme in $C$. By Lemma 3.4 all measures in $C$ possess a common zero on $\partial R$, say $P$. If $m_{1}$ were not extreme in $\Re_{a}$, then there would exist $m_{2}, m_{3} \in \mathfrak{R}_{a}$, $m_{2} \neq m_{3}$, such that $m_{1}=\left(m_{2}+m_{3}\right) / 2$. Then $m_{2}(P)=m_{3}(P)=0$, and by maximality, $m_{2}$ and $m_{3} \in C$, which implies $m_{1}$ is not extreme in $C$, a contradiction.

Since $C$ is the closed convex hull of its extreme points and $C \cap S$ is nonempty, it follows that $\mu$ is an extreme point of $C$. Thus $\partial \Re_{a} \cap S \subset \cup_{K \in \mathcal{C}} K$, where $\mathcal{C}$ is the class of maximal convex subsets of $\partial \Re_{a}$ which contain $\mu$. By Lemma 3.5 it follows that $\mathcal{C}$ has at most $r$ elements. Hence, since $\partial\left(\Re_{a} \cap S\right)=\left(\partial \Re_{a} \cap S\right)$ $\cup\left(\partial S \cap \Re_{a}\right)$ and since $\partial S \cap \pi_{a}$ is clearly a maximal convex subset of $\partial\left(\Re_{a} \cap S\right)$, we see that $\partial\left(\Re_{a} \cap S\right)$ has at most $r+1$ maximal convex subsets. Now Lemma 3.6 shows that $\Re_{a} \cap S$ is an $r$-simplex.

By the preceding it follows that $\mu$ is an intersection of $r$ hyperplanes, $P_{1}, \ldots, P_{r}$ each of which contains a face of $\Re_{a}$, and these $P_{i}$ 's determine $r$ affinely independent line segments, $E_{1}, \ldots, E_{r}$ which are one dimensional edges in $\partial \Re_{a}$ with $\mu$ as a common endpoint. (The case $r=3$ may prove illuminating.) Furthermore, the interior of the convex hull of every $r-1 E_{i}$ 's is contained in a face. If $m_{1}$ and $m_{2}$ belong respectively to two distinct faces, then by Lemma 3.1 it follows that $\left[m_{1}, m_{2}\right]$ is not contained in $\partial \pi_{a}$. Hence $m_{1}$ and $m_{2}$ have no common zeros. It follows that each measure $\mu_{i} \in E_{i}$ has at least $r-1$ distinct zeros on $\partial R$, and these, of course, must also be zeros of $\mu$. If $\mu_{i} \neq \mu$, then $\mu_{i}$ has precisely $r-1$ zeros in common with $\mu$. This implies that if $\mu_{i} \in$ interior of $E_{i}$ and $\mu_{j} \in$ interior of $E_{j}, i \neq j$, then $\mu_{i}$ and $\mu_{j}$ cannot have precisely the same zeros in common with $\mu$. Consequently, $\mu$ must have $r$ distinct zeros on $\partial R$. These zeros must be double by Theorem 2.4 .

Choose an $m$ from the interior of any $E_{i}$. Then $m$ has $r-1$ double zeros on $\partial R$ and one simple zero in $R$, for if $m$ had all of its $r$ zeros on $\partial R$, it would be extreme by Corollary 3.3 .

The next lemma is due to Gamelin and Voichick (see [7, p. 924]). 
Lemma 3.8. If a function $h$ is meromorphic on $\bar{R}$, real on $\partial R$ and has just one pole, a simple pole, in $R$, then $h$ is a conformal homeomorphism of $R$ onto $S^{2}$ minus a finite number of slits contained in $\mathrm{Re}$.

Theorem 1.1 now follows if we take $\mu$ and $m$ as in Lemma 3.7 and set $h=\mu / m$ in Lemma 3.8.

We next discuss an example in which $\mathfrak{T}_{a}$ has isolated extreme points.

Let $R_{r}$ be any bounded region in $\mathbf{C}$ which is bounded by $r+1$ disjoint circles, $C_{0}, C_{1}, \ldots, C_{r}$ with respective centers $a_{0}, a_{1}, \ldots, a_{r}$ on the real axis $\operatorname{Re}$. We shall assume $a_{1}<a_{2}<\cdots<a_{r}$ and that $C_{0}$ is the boundary of the unbounded component of $\mathbf{C} \backslash R_{r}$. Let $C_{0} \cap \operatorname{Re}=\left\{a_{-1}, a_{r+1}\right\}$ with $a_{-1}<a_{r+1}$.

Theorem 3.9. If $a \in R_{r} \cap \operatorname{Re}$, then $\Re_{a}$ has $2 r$ faces and at most $2^{r}$ extreme points.

Remarks. We shall see that $\mathfrak{T}_{a}$ has four extreme points when $a \in R_{2} \cap$ Re. A somewhat tedious exercise shows that $\Re_{a}$ has eight extreme points for $a \in R_{3} \cap \mathrm{Re}$. It would be interesting to know if $\mathfrak{T}_{a}$ has precisely $2^{r}$ extreme points for all $r$ when $a \in R_{r} \cap \operatorname{Re}$. Before giving a proof of Theorem 3.9, we shall present some relevant material.

Let $\Omega_{j}$ be harmonic measure for $C_{j}, 1 \leq j \leq r$, i.e., $\Omega_{j}$ is the solution to the Dirichlet problem for $\bar{R}_{r}$ with boundary values 1 on $C_{j}$ and 0 on $C_{k}, j \neq k$. A basis for $V$ is given by $* d \Omega_{j}-i d \Omega_{j}, 1 \leq j \leq r$, and since each $\Omega_{j}$ is symmetric with respect to $\mathrm{Re}$, elements of $V$ must also possess this symmetry. The next lemma follows easily.

Lemma 3.10. (i) If $\eta \not \equiv$ is in $V$ and has a zero in $\partial R_{r} \cap \operatorname{Re}$, then this zero has even order.

(ii) If $\eta$ is in $V$ and has a zero $z \in \partial R_{r}$, then it has a zero at $\bar{z}$ of the same order.

The next two lemmas hold for arbitrary $R$.

Lemma 3.11. Suppose $z_{1}, \ldots, z_{q} \in R$ and $q<r / 2$. Then there is a nonzero $\eta \in V$ with zeros at $z_{i}, 1 \leq i \leq q$.

Proof. Let $\omega$ be a nowhere zero holomorphic differential on $\bar{R}$ and $\omega_{i}$, $1 \leq i \leq r$, be a basis of Schottky differentials for $V$. Define $Q_{i}$ by $\omega_{i}=Q_{i} \omega$, $1 \leq i \leq r$, and let $v_{i}=\left(Q_{i}\left(z_{1}\right), \ldots, Q_{i}\left(z_{q}\right)\right), 1 \leq i \leq r$. Each $v_{i}$ can be viewed as an element of complex $q$ space. Since the number of $v_{i}$ 's is $r$ and $2 q<r$, it follows that there exist $\alpha_{1}, \ldots, \alpha_{r} \in \operatorname{Re}$, not all zero, such that $\sum_{i=1}^{r} \alpha_{i} v_{i}=0$. The differential $\eta=\sum_{i=1}^{r} \alpha_{i} \omega_{i}$ satisfies the conclusion of the lemma.

Lemma 3.12. Let $z_{1}, \ldots, z_{k}$ be distinct points on $\partial R$ and $q_{1}, \ldots, q_{k}$ be positive integers whose sum is less than $r$. Then there is a nonzero $\eta \in V$ such that $\eta$ has $a$ zero at each $z_{i}$ of at least order $q_{i}$.

A proof of Lemma 3.12 for planar $R$, which modifies easily for general $R$, can be found in [12, p. 375]. We shall need Lemma 3.12 for planar $R$ only. 
Lemma 3.13 Let $a \in R_{r} \cap \operatorname{Re}$ and suppose, without loss of generality, that $a \in R_{r} \cap\left(a_{1}, a_{2}\right)$. Then each $\mu \in \mathfrak{R}_{a}$ has a zero on each of the $r$ components of $\bar{R}_{r} \cap \operatorname{Re} \backslash\left(a_{1}, a_{2}\right)=Y$. We shall denote this property by $\psi$ and let the components of $Y$ be given by $\left[c_{1}, c_{1}^{\prime}\right], \ldots,\left[c_{r}, c_{r}^{\prime}\right]$.

Proof. Let $d m_{a}=* d g /-2 \pi=(-1 / 2 \pi)(\partial g / \partial n) d \sigma$ be harmonic measure, where $\partial / \partial n$ denotes differentiation along the outer unit normal to $\partial R_{r}$ and $\sigma$ denotes arc length. Since $g(z, a)$, the Green's function for $\bar{R}_{r}$ with singularity $a$, vanishes at each boundary point of $Y$, the interior of each $\left[c_{i}, c_{i}^{\prime}\right]$ contains at least one zero of $\partial g / \partial x$. The function $\partial g / \partial y$ is zero on $R_{r} \cap \operatorname{Re}$ since $g(z, a)$ is symmetric about Re; therefore, $m_{a}$ has property $\psi$. Let $\mu$ be any element of $\mathfrak{R}_{a}$ and consider the homotopy

$$
\mu_{t}=t m_{a}+(1-t) \mu, \quad 0 \leq t \leq 1
$$

Let $U=\left\{t \in[0,1] \mid \mu_{t}\right.$ has property $\left.\psi\right\}$. Then $U$ is nonempty since $1 \in U$. If $t_{0} \in U$, then for $t$ sufficiently near $t_{0}, \mu_{t}$ has a zero $P_{j}$ in each of the $r$ open strips $\left\{x+y i \mid \alpha_{j}<x<\alpha_{j+1},-\infty<y<+\infty\right\}, j=-1,2,3, \ldots, r$. The measure $m_{a}$ is symmetric with respect to Re; hence, any $\mu_{t}$, being the sum of $m_{a}$ and an element of $V$, is symmetric with respect to Re. Since no $\mu_{t}$ can have more than $r$ zero points, the $r P_{j}^{\prime}$ 's must therefore lie on Re. The set $U$ is thus open. It is easy to see that $U$ is closed. Consequently, $U=[0,1]$ and $\mu_{0}=\mu$ has property $\psi$.

Lemma 3.14. If $\mu \in \mathfrak{T}_{a}, a \in Y \cap R_{r}$ is extreme, then it must have all its zeros in $\partial R_{r} \cap \mathrm{Re}$.

Proof. If not, then by Lemma 3.12 and Lemma 3.10(i) it is easy to produce a nonzero $\eta \in V$ such that $\eta$ has a zero at each zero of $\mu$ on $\partial R_{r} \cap \operatorname{Re}$ of at least as high order. The measure $\mu$ would then fail to be extreme by Lemma 3.2.

Lemma 3.14 shows that the zeros of each extreme point of $\pi_{a}$ must lie in the finite set $\partial Y$; consequently, $\Re_{a}$ has only finitely many extreme points and Lemma 3.7 implies each extreme point has $r$ double zeros on $\partial R$. These remarks and Lemma 3.13 show that $\mathfrak{R}_{a}$ has at most $2^{r}$ extreme points.

Let $\mu \in \mathfrak{N}_{a}$ be extreme. Without loss of generality we can assume $\mu$ has zeros at $c_{1}, c_{2}, \ldots, c_{r}$. The measure $\mu$ is in the intersection of the closure of some $r$ faces. Each face must have elements with at least one zero in common with $\mu$. There are $r$ possible zeros, $c_{1}, c_{2}, \ldots, c_{n}$ and by Lemma 3.1, no two faces can have elements possessing more than one of these zeros. Consequently, each zero, $c_{1}, c_{2}, \ldots, c_{n}$ corresponds to one and only one face. We claim that given any $z \in \partial Y$, there is an $m \in \partial \Re_{a}$ with a zero at $z$. The notation will be that of Lemma 3.7. Each $E_{i}$ must have an extreme point $\mu_{i}$ with precisely $r-1$ zero points in common with $\mu$. If, say, the zero set of $\mu_{i}$ is $\left\{c_{1}, c_{2}, \ldots, c_{k-1}, c_{k}^{\prime}, c_{k+1}, \ldots\right.$, $\left.c_{r}\right\}$ and the zero set of $\mu_{j} \in E_{j}$ is $\left\{c_{1}, c_{2}, \ldots, c_{n-1}, c_{n}^{\prime}, c_{n+1}, \ldots, c_{r}\right\}, i \neq j$, then $c_{k}^{\prime}$ and $c_{n}^{\prime}$ cannot lie in the same component of $Y$. If they did, then either $c_{k}^{\prime}=c_{q}$, 
or $c_{n}^{\prime}=c_{q}$ for some $q, 1 \leq q \leq r$. Then either the zeros of $\mu$ and $\mu_{i}$, or the zeros of $\mu$ and $\mu_{j}$ coincide, a contradiction. The claim follows easily.

Each $z \in \partial Y$ must therefore be a zero of an extreme point and can correspond to one and only one face. The set $\partial Y$ has $2 r$ elements, so $\Re_{a}$ must have $2 r$ faces. The proof of Theorem 3.9 is now complete.

Corollary 3.15. If $a \in R_{2} \cap \mathrm{Re}$, then $\Re_{a}$ has precisely four extreme points.

Let $R$ be arbitrary, $W=\{z \in \partial R \mid$ no element of $V$ has a simple zero at $z\}$ and $Z=\left\{z \in \partial R \mid\right.$ there is an $m \in \Re_{a}$ with a zero at $\left.z\right\}$.

Theorem 3.16. If $a \in R$, then the number of faces of $\mathfrak{T}_{a} \leq$ the cardinality of $W \cap Z$.

Proof. If $\mathfrak{T}_{a}$ has no faces, then Theorem 3.16 is trivially true. Otherwise, let $F$ be a face. Then $F$ is $r-1$ dimensional and is contained in an $r-1$ dimensional hyperplane. Since the zero measure is not in $F$, there are $r$ elements in $F$, $\mu_{1}, \ldots, \mu_{r}$ such that $\mu_{i}-\mu_{1}, 2 \leq i \leq r$, are linearly independent. Since the $\mu_{i}$ 's belong to a face, they must have at least one common zero, say $z$, which is of at least order two and, of course, $z \in Z$. It is easy to find an $\eta \in V$ which is nowhere zero on $\partial R$ (see, e.g., [1, pp. 4-5]). Then $\left\{\mu_{i}-\mu_{1} \mid 2 \leq i \leq r\right\} \cup\{\eta\}$ is a basis for $V$; consequently, $z \in W$. Lemma 3.1 implies no two faces can have common elements, and Theorem 3.16 follows.

It is easy to show using Lemmas 3.12 and 3.10 that for $z \in \partial R_{r} \backslash \operatorname{Re}$ there is an $\eta \in V$ with a simple zero at $z$ and that $W \cap Z=Y$ for this case. Lemma 3.14 then shows that Theorem 3.16 gives the best possible estimate of the number of faces. It is not known yet whether equality always holds in Theorem 3.16.

Lemma 3.17. If $a \in R_{r} \backslash \operatorname{Re}$ and $r \geq 3$, then no $\mu$ in $\mathfrak{N}_{a}$ can have $r$ double zeros on $\partial R \cap$ Re.

Proof. Suppose there is such a $\mu$. We have $\mu=m_{a}+\eta$ for some $\eta \in V$. The measure $d m_{a}$ can be expressed by $(-1 / 2 \pi)(\partial g / \partial n)(z, a) d \sigma$ on $\partial R$ (see Lemma 3.13), and it is easy to verify that harmonic measure for $e_{a}$ is given on $\partial R$ by $d m_{\bar{a}}=(-1 / 2 \pi)(\partial g / \partial n)(\bar{z}, \bar{a}) d \sigma$. It follows from our hypothesis that each zero $z_{i}$ of $\mu$ on $C_{i}$ is real. In some neighborhood of $z_{i}$ on $C_{i}$ there are coordinates such that the following representations hold (the $a_{i j}$ 's and $b_{i}$ 's are certain real numbers):

$$
\begin{aligned}
d m_{a}\left(\theta_{i}\right) & =\left(a_{i 0}+a_{i 1} \theta_{i}+a_{i 2} \theta_{i}^{2}+\cdots\right) d \theta_{i}, \\
d \eta\left(\theta_{i}\right) & =\left(-a_{i 0}-a_{i 1} \theta_{i}+b_{i} \theta_{i}^{2}+\cdots\right) d \theta_{i} \text { and } \\
d m_{\bar{a}}\left(\theta_{i}\right) & =d m_{a}\left(-\theta_{i}\right)=\left(a_{i 0}-a_{i 1} \theta_{i}+a_{i 2} \theta_{i}^{2}+\cdots\right) d \theta_{i},
\end{aligned}
$$

where $\theta_{i}=0$ corresponds to $z_{i}$. Then $d \mu\left(\theta_{i}\right)=\left[\left(a_{i 2}+b_{i}\right) \theta_{i}^{2}+\cdots\right] d \theta_{i}$ and $\mu_{1}$ defined by $\mu_{1}=m_{\bar{a}}+\eta$ satisfies $d \mu_{1}\left(\theta_{i}\right)=\left[\left(a_{i 2}+b_{i}\right) \theta_{i}^{2}+\cdots\right] d \theta_{i}$. Let $\nu=\mu$ $-\mu_{1}$. Then $\nu$ is real on $\partial R$ and has a zero of at least order three at each of the $r$ zero points of $\mu$. Therefore, $\nu$ has at least $3 r$ zeros on $\partial R$ if $r$ is even, and since $\nu$ 
must have an even number of zeros on $\partial R$, it has at least $3 r+1$ zeros on $\partial R$ if $r$ is odd. The measure $\nu$ has just two poles, simple poles at $a$ and $\bar{a}$.

The argument principle shows that if $n=$ the number of zeros of $\nu$ minus its number of poles, where zeros on $\partial R$ are counted with one-half their multiplicities, then $n=r-1$. The considerations of the preceding paragraph yield a contradiction for $r \geq 3$.

Theorem 3.18. If $r \geq 2$ and $a \in R_{r} \backslash R e$, then $\Re_{a}$ has no isolated extreme points.

Proof. Suppose $\pi_{a}$ has an isolated extreme point $\mu$. Then, by Lemma 3.7, $\mu$ has double zeros on $\partial R$. Refer to the proof of Lemma 3.7. If $\mu_{i}$ and $\mu_{j}$ belong to the interiors of $E_{i}$ and $E_{j}$ respectively, $i \neq j$, then each has precisely $r-1$ double zeros in common with $\mu$. The measures $\mu-\mu_{i}$ and $\mu-\mu_{j} \in V$, and each has precisely $r-1$ double zeros on $\partial R$. Elements of $V$ are symmetric with respect to $\operatorname{Re}$ and can have no more than $r-1$ double zeros; therefore, $\mu-\mu_{i}$ and $\mu-\mu_{j}$ each has precisely $r-1$ double zeros in $\partial R \cap \operatorname{Re}$. The zero sets of $\mu_{i}$ and $\mu_{j}$, and thus those of $\mu-\mu_{i}$ and $\mu-\mu_{j}$, are not equal for $i \neq j$. It follows that $\mu$ has $r$ double zeros on $\partial R \cap \operatorname{Re}$. A contradiction results from Lemma 3.17 if $r \geq 3$. The case $r=2$ is covered by Theorem 1.2.

Theorem 3.19. If $r \geq 3$ and is odd and $a \in R_{r}$, then $\mathfrak{R}_{a}$ is not strictly convex.

Proof. Let $m_{a}$ have zeros $z_{1}, \ldots, z_{r}$ in $R_{r}$ (see Theorem 2.3). Lemma 3.11 gives a nonzero $\eta \in V$ with zeros at $z_{1}, \ldots, z_{(r-1) / 2}$. For some $\varepsilon, m_{a}+\varepsilon \eta=m$ $\in \partial \Re_{a}$, and since $m$ has zeros at $z_{1}, \ldots, z_{(r-1) / 2}, m$ can have no more than $r+1$ zeros on $\partial R_{r}$ counting multiplicities. The zeros on $\partial R_{r}$ are of even order, so if $m$ has less than $r+1$ zeros on $\partial R_{r}$ it has at most $r-1$. Then Lemmas 3.12 and 3.2 imply $m$ is not extreme.

Suppose $m$ has $r+1$ zeros on $\partial R_{r}$. Let $P_{1}, \ldots, P_{n}$ be the zero points of $m$ on $\partial R_{r}$ with respective multiplicities $2 v_{i}, v_{i}$ a positive integer. Using Lemmas 3.12 and 3.10 we can find an $\eta \in V$ which has zeros of order $2 v_{i}$ at $P_{i}, 1 \leq i \leq n-1$, and either no zero or a zero of even order at $P_{n}$. In both cases for some $\varepsilon>0$, we have $m+\delta \eta \in \partial \Re_{a}$ for either $0 \leq \delta<\varepsilon$ or $-\varepsilon<\delta \leq 0$. Consequently, $\partial \Re_{a}$ contains a line segment and $\Re_{a}$ is not strictly convex.

It is conjectured that Theorem 3.19 is true for $r$ even and greater than two. Theorem 1.2, which we prove next, shows Theorem 3.19 is false for $r=2$.

Proof of Theorem 1.2. Let $R$ be such that $g=0$ and $s=3$. Then it is easy to show that $R$ is conformally equivalent to some $R_{2}$, so without loss of generality for this part of the proof we can and shall assume $R=R_{2}$.

Let $a \in R_{2}$ and suppose $\Re_{a}$ is not strictly convex. Then $\partial \mathscr{T}_{a}$ contains an open line segment $E$. Let $\mu \in E$. Then $\mu$ has at least one double zero $z$ on $\partial R_{2}$ since $\mu \in \partial \Re_{a}$. If it had two distinct zeros on $\partial R$, it would be extreme by Corollary 3.3. Therefore, $\mu$ has a simple zero at some $b \in R_{2}$. Let $\mu_{1} \in E$ and $\mu_{1} \neq \mu$. Then $\mu_{1}(z)=0$ and $\eta$ defined by $\mu_{1}-\mu$ is a nonzero element of $V$.

By Lemma 3.8 the function $h=\eta / \mu$ is a conformal homeomorphism of $R_{2}$ onto a domain $D_{1}$ in $S^{2}$ such that $\partial D_{1}$ consists of three slits in Re. It is easy to 
check that the class of real valued functions which are harmonic on $D_{1}$ and constant on each slit is carried by $h^{-1}$ onto the class of real valued functions which are harmonic on $R_{2}$, continuous on $\bar{R}_{2}$ and constant on each component of $\partial R_{2}$. The first order complex differentials $* d \Omega-i d \Omega$ for $\Omega$ in these classes have one zero point each, so by symmetry these points must lie on Re. It is easy to verify that each element of $D_{1} \cap \operatorname{Re}$ and $R_{2} \cap \operatorname{Re}$ is a zero of some differential of the type mentioned above; consequently, $h^{-1}\left(D_{1} \cap \mathrm{Re}\right)=R_{2}$ $\cap$ Re. Hence $a \in \operatorname{Re}$ since $h(a)=0$. Corollary 3.13 shows that $\mathfrak{T}_{a}$ has precisely four extreme points for any $a$ on the three segments which comprise $R_{2} \cap$ Re.

Now suppose $g=1$ and $s=1$. If $\mathscr{R}_{a}$ is assumed to be not strictly convex, then by Lemma 3.2 there is some $\eta \in V$ with a double zero $z$ on $\partial R$. We have $r=2$, which implies $\eta$ has no other zeros. Hence, since $\partial R$ has only one component, $\eta$ is either strictly positive or strictly negative on $\partial R \backslash\{z\}$. But then $\eta$ would fail to annihilate $A$. Thus $\mathfrak{R}_{a}$ is strictly convex and the proof of Theorem 1.2 is complete.

\section{BIBLIOGRAPHY}

1. P. R. Ahern, On the geometry of the unit ball in the space of real annihilating measures, Pacific J. Math. 28 (1969), 1-7. MR 40 \#7785.

2. P. R. Ahern and D. Sarason, The $H^{p}$ spaces of a class of function algebras, Acta Math. 117 (1967), 123-163. MR 36 \#689.

3. L. Ahlfors, Open Riemann surfaces and extremal problems on compact subregions, Comment. Math. Helv. 24 (1950), 100-134. MR 12, 90; 13, 1138.

4. L. Ahlfors and L. Sario, Riemann surfaces, Princeton Math. Series, no. 26, Princeton Univ. Press, Princeton, N.J., 1960. MR 22 \#5729.

5. L. Bieberbach, Conformal mapping, Chelsea, New York, 1953. MR 14, 462.

6. H. Busemann, Convex surfaces, Interscience Tracts in Pure and Appl. Math., no. 6, Interscience, New York, 1958. MR 21 \#3900.

7. T. W. Gamelin and M. Voichick, Extreme points in spaces of analytic functions, Canad. J. Math. 20 (1968), 919-928. MR 37 \#3023.

8. K. deLeeuw and W. Rudin, Extreme points and extremum problems in $H_{1}$, Pacific J. Math. 8 (1958), 467-485. MR 20 \#5426.

9. Z. Nehari, Conformal mapping, McGraw-Hill, New York, 1952. MR 13, 640.

10. A. H. Read, A converse to Cauchy's theorem and applications to extremal problems, Acta Math. 100 (1958), 1-22. MR 20 \#4640.

11. H. L. Royden, The boundary values of analytic and harmonic functions, Math. Z. 78 (1962), 1-24. MR 25 \#2190.

12. D. Sarason, Representing measures for $R(X)$ and their Green's functions, J. Functional Analysis 7 (1971), 359-385.

13. G. Springer, Introduction to Riemann surfaces, Addison-Wesley, Reading, Mass., 1957. MR 19, 1169.

14. M. Voichick and L. Zacman, Inner and outer functions on Riemann surfaces, Proc. Amer. Math. Soc. 16 (1965), 1200-1204. MR 32 \#1359.

15. J. Wermer, Analytic disks in maximal ideal spaces, Amer. J. Math. 86 (1964), 161-170. MR 28 \#5355.

Department of Mathematics, University of California, Berkeley, California 94720

Current address: General Motors Research Laboratories, Transportation and Urban Analysis Department, Warren, Michigan 48090 